\title{
Slow Dynamics Near Jamming
}

\author{
Kuniyasu Saitoh, Vanessa Magnanimo and Stefan Luding \\ Faculty of Engineering Technology, University of Twente, Enschede, the Netherlands
}

\begin{abstract}
Static and dynamic properties of two-dimensional bidisperse dissipative particles are numerically studied near the jamming transition. We investigate the dependency of the critical scaling on the ratio of the different diameters and find a new scaling of the maximum overlap (not consistent with the scaling of the mean overlap). The ratio of kinetic and potential energies drastically slows down near the jamming transition, i.e. the relaxation time diverges at the jamming point.
\end{abstract}

Keywords: jamming transition, polydispersity, critical slowing down

PACS: 45.70.-n, 45.70.Cc, 61.43.-j

\section{INTRODUCTION}

Jamming is an universal feature of both thermal and athermal systems, for instance, glasses, granular particles, emulsions, colloidal suspensions, foams, etc. where constituents are arrested in disordered states so that the material obtains rigidity. The jamming transition is governed by temperature, density and external loads, and a lot of systems can be mapped onto a unified phase diagram $[1,2,3]$.

Jamming of athermal systems, i.e. granular particles [4, 5, 6, 7], emulsions [8, 9] and foams [10, 11], occurs at zero temperature at the critical density (area fraction) $\phi_{c}$ [1]. At this point, each particle begins to touch its neighbors and the mean coordination number $z$, defined as the average number of contacts per particle, jumps from zero to the isostatic value $z_{c}=2 d$ in $d$-dimensions [12]. Some macroscopic variables also indicate the acquisition of rigidity, for example, the pressure $p$ and the shear modulus $G$ start to increase from zero, and the bulk modulus $K$ discontinuously jumps to a non-zero value $[4,5,6,7]$. Above this threshold, the excess coordination number $\Delta z=z-z_{c}, p, G$ and $K$ respectively scale as $\Delta z \sim \Delta \phi^{1 / 2}, p \sim \Delta \phi^{\psi}, G \sim \Delta \phi^{\gamma}$ and $K \sim \Delta \phi^{\lambda}$ with the distance from the jamming point $\Delta \phi=\phi-\phi_{c}$. In the case of monodisperse particles, the first peak of the radial distribution function $g_{1}$ scales as $g_{1} \sim \Delta \phi^{-1}[13,14,15,16]$. In the case of bidisperse particles, one can also see a similar divergence of the radial distribution function with the scaled distance [7]. Moreover, the mean overlap between particles $\langle\boldsymbol{\delta}\rangle$ linearly scales as $\langle\delta\rangle \sim \Delta \phi$.

Those power law scalings are analogous to those found in critical phenomena. However, some variables show discontinuous changes at the critical point as in first-order phase transitions. Furthermore, the critical exponents $\psi, \gamma$ and $\lambda$ depend on the interparticle forces, which suggests that the jamming transition is entirely different from usual critical phenomena $[4,5,6,7]$.

Even though the critical scalings above the jamming transition are well established, not much attention has been paid to the critical amplitudes. Many previous works on bidisperse systems only focused on the particular case that the ratio of the different diameters equals $\rho=1$.4. Furthermore, the dynamic properties are not understood yet.

In this paper, we study the static and dynamic properties of two-dimensional bidisperse particle systems by numerical simulations and investigate the dependency of the critical amplitudes on $\rho$. We also study the dynamic properties near the jamming transition, where we adopt the ratio of kinetic to potential energies to quantify relaxation and show its drastic slowing down near the jamming point.

\section{MOLECULAR DYNAMICS SIMULATION}

We investigate two-dimensional packings of bidisperse dissipative particles using molecular dynamics (MD) simulations. Our strategy is to increase the diameter of each particle until a desired area fraction $\phi$ is obtained. After the desired area fraction is obtained, the kinetic energy decreases to zero due to the dissipative forces between particles and the system relaxes to its static state. We study dynamic properties during the relaxation and static properties after the relaxation. 
At first, we prepare a binary mixture of large and small particles with initial diameters $\sigma_{\mathrm{L}}(0)$ and $\sigma_{\mathrm{S}}(0)$, respectively, where the total number of particles is $N=32768$. We randomly distribute them in a $L \times L$ periodic box one-by-one, avoiding overlap between particles. Then, we slowly increase the diameter of each particle $[17,18,19,20]$ as:

$$
\dot{\sigma}_{a}(t)=g_{r} \sigma_{a}(0) \quad(a=\mathrm{L} \text { or S })
$$

with a constant growth rate $g_{r}$. Because we fix the mass density of particles, the mass also increases while we increase the diameter $\sigma_{a}(t)=\sigma_{a}(0)\left(g_{r} t+1\right)$. Therefore, the size ratio $\rho \equiv \sigma_{\mathrm{L}}(t) / \sigma_{\mathrm{S}}(t)=\sigma_{\mathrm{L}}(0) / \sigma_{\mathrm{S}}(0)$ does not change throughout our simulations.

Each particle $i$ can be in contact with several other particles $j$ and moves according to the equation of motion

$$
m_{i}(t) \ddot{\mathbf{x}}_{i}=\sum_{j}\left\{k_{n} \delta_{i j}-\eta_{n}\left(\mathbf{v}_{i j} \cdot \mathbf{n}_{i j}\right)\right\} \mathbf{n}_{i j}
$$

where $m_{i}(t), k_{n}, \eta_{n}, \delta_{i j}, \mathbf{n}_{i j}=\left(\mathbf{x}_{i}-\mathbf{x}_{j}\right) /\left|\mathbf{x}_{i}-\mathbf{x}_{j}\right|$, and $\mathbf{v}_{i j}=\dot{\mathbf{x}}_{i}-\dot{\mathbf{x}}_{j}$ are the mass of particle $i$, the spring constant, the viscosity coefficient, the overlap, the normal unit vector, and the relative velocity, respectively. The vector $\mathbf{x}_{i}$ is the position of particle $i$, and each dot above $\mathbf{x}$ represents the time derivative.

When the area fraction reaches the desired value $\phi$ at $t=t_{0}$, we stop to increase the diameter of each particle. Then, the kinetic energy of the system decreases due to the dissipative forces and we assume the system is relaxed to its static state if the ratio of kinetic to potential energies becomes lower than $10^{-6}$. In the following, we scale mass, length and time by $m_{u}=m_{\mathrm{L}}\left(t_{0}\right), l_{u}=\sigma_{\mathrm{L}}\left(t_{0}\right)$ and $t_{u}=m_{\mathrm{L}}\left(t_{0}\right) / \eta_{n}$, respectively, and use $g_{r}=10^{-4} / t_{u}, k_{n}=4.0 \times 10^{5} m_{u} / t_{u}^{2}$ and $\eta_{n}=m_{u} / t_{u}$.

From Eq. (2), the restitution coefficient in the relaxation stage $\left(t>t_{0}\right)$ defined as the ratio of speeds after and before a collinear collision is calculated as [21]

$$
e_{n}=e^{-\eta_{0} t_{c}}
$$

where $t_{c}=\pi / \sqrt{\left(k_{n} / m_{i j}\right)-\eta_{0}^{2}}$ and $\eta_{0}=\eta_{n} /\left(2 m_{i j}\right)$ are a typical response time and the rescaled viscosity coefficient with the reduced mass $m_{i j}=m_{i}\left(t_{0}\right) m_{j}\left(t_{0}\right) /\left(m_{i}\left(t_{0}\right)+m_{j}\left(t_{0}\right)\right)$. Although the restitution coefficients between two small particles, two large particles, and small and large particles are slightly different, we find $e_{n}=0.99 \pm 0.003$ in the three cases since dissipation is rather weak.

\section{CRITICAL SCALING}

Changing the values of $\phi$ and $\rho$ between $0.8 \leq \phi \leq 0.9$ and $1.2 \leq \rho \leq 2.4$, respectively, we repeat our simulations and measure the mean overlap $\langle\delta\rangle$, the mean coordination number $z$, the pressure $p$, the first peak of the radial distribution function with the scaled distance $g_{+}$and the maximum overlap $\delta_{\mathrm{m}}$, after each system is relaxed to its static state. We define the critical area fraction $\phi_{c}$ as the point at which $\langle\delta\rangle$ starts to increase from zero and $z$ jumps from zero to the isostatic value $z_{c}=4$. It should be noted that we remove rattlers of which the number of contacts are less than 3 from the statistics, because they do not contribute to the contact- and force-chain networks $[4,5,6,7]$.

The critical area fraction depends on both $\rho$ and $g_{r}$. Figure 1 shows $\phi_{c}$ as a function of the relative standard deviation

$$
R \equiv \frac{\sqrt{\left\langle\sigma^{2}\right\rangle-\langle\sigma\rangle^{2}}}{\langle\sigma\rangle}
$$

where $\langle\sigma\rangle=(1+\rho) \sigma_{\mathrm{S}}\left(t_{0}\right) / 2$ and $\left\langle\sigma^{2}\right\rangle=\left(1+\rho^{2}\right) \sigma_{\mathrm{S}}\left(t_{0}\right)^{2} / 2$ are the mean diameter and the mean square of diameter, respectively. In this figure, the closed circles are our results and the open circles are the results of another simulation of bidisperse hard spheres in two dimensions [22], where a large discrepancy between these results can be seen below $R=0.2$, mainly caused by the dependency of $\phi_{c}$ on $g_{r}$. In this paper, we do not discuss the rate dependency of $\phi_{c}$, however, it is known that $\phi_{c}$ strongly depends on $g_{r}$, if $R$ is small (see Ref. [23] for a more detailed discussion). We also list our results of $\phi_{c}$ for different $\rho$ and $R$ in Table 1 .

Above $\phi_{c},\langle\delta\rangle$ scales as $[4,5,6,7]$

$$
\langle\delta\rangle=B_{\delta}(\rho) \Delta \phi^{\mu},
$$

where $\mu$ and $B_{\delta}(\rho)$ are the critical exponent and the critical amplitude, respectively (see Table 1). From our simulations, we find $\mu \simeq 1.0$, i.e. $\langle\delta\rangle$ depends linearly on $\Delta \phi$ (see Table 2 ). 


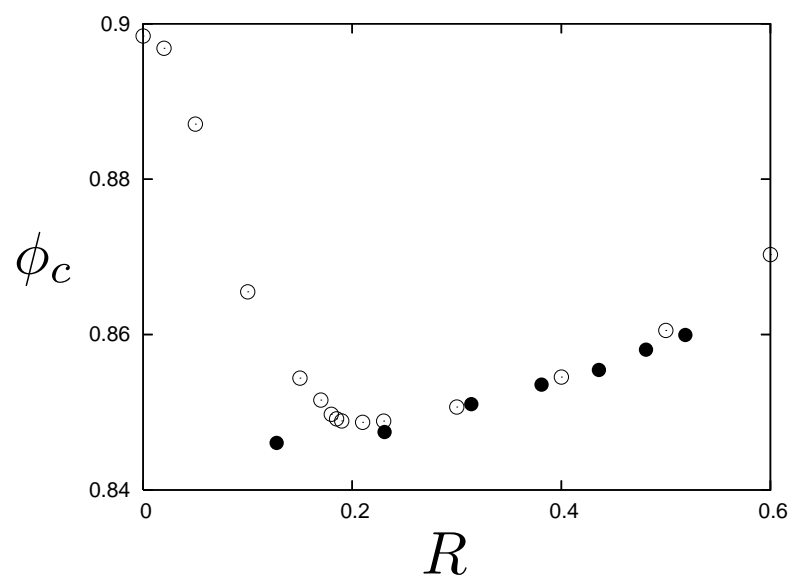

FIGURE 1. Critical area fraction $\phi_{c}$ as a function of $R$, where the closed circles are the results of our simulations and the open circles are the results of another simulation of bidisperse hard spheres in two dimensions [22].

TABLE 1. The critical area fraction and the critical amplitudes.

\begin{tabular}{ccccccccc}
\hline$\rho$ & $R$ & $\phi_{c}$ & $B_{\delta}$ & $A_{p} / k_{n}$ & $A_{z}$ & $A_{+}$ & $A_{\mathrm{m}}$ & $A_{\mathrm{m}} / B_{\delta}$ \\
\hline 1.2 & 0.127 & 0.846054 & 0.262691 & 0.062944 & 9.623507 & 0.108473 & 3.271311 & 12.453076 \\
1.4 & 0.230 & 0.847454 & 0.247794 & 0.062175 & 9.789582 & 0.112457 & 3.839339 & 15.494075 \\
1.6 & 0.313 & 0.851053 & 0.234816 & 0.058778 & 9.696656 & 0.107054 & 4.508161 & 19.198696 \\
1.8 & 0.381 & 0.853553 & 0.240875 & 0.061023 & 10.680258 & 0.102261 & 4.554234 & 18.907043 \\
2.0 & 0.435 & 0.855449 & 0.246302 & 0.062333 & 9.661944 & 0.109497 & 4.378124 & 17.775430 \\
2.2 & 0.481 & 0.858054 & 0.278428 & 0.067636 & 9.986629 & 0.122986 & 5.144424 & 18.476676 \\
2.4 & 0.518 & 0.859950 & 0.268369 & 0.073998 & 10.649745 & 0.207261 & 5.304872 & 19.767081 \\
\hline
\end{tabular}

We define the pressure as the virial pressure $p=\sum_{i<j} r_{i j} f_{i j} / L^{2}$ with the interparticle distance $r_{i j}$ and force $f_{i j}$ [24] and introduce the excess coordination number as $\Delta z \equiv z-z_{c}$. Because we use bidisperse particles, the usual radial distribution function has three peaks around $\sigma_{\mathrm{S}}, \sigma_{\mathrm{L}}$ and $\left(\sigma_{\mathrm{L}}+\sigma_{\mathrm{S}}\right) / 2$, respectively. However, if we introduce the scaled distance between particles $i$ and $j$ as $\xi=r_{i j} /\left(r_{i}+r_{j}\right)$, where $r_{i}$ and $r_{j}$ are the radii of particles $i$ and $j$, respectively, the radial distribution function of $\xi$ has the first peak $g_{+}$around $\xi=1$. Then, we find $p, \Delta z$ and $g_{+}$scale as

$$
\begin{aligned}
p & =A_{p}(\rho)\langle\delta\rangle^{\psi}, \\
\Delta z & =A_{z}(\rho)\langle\delta\rangle^{\zeta}, \\
g_{+} & =A_{+}(\rho)\langle\delta\rangle^{-\eta_{+}},
\end{aligned}
$$

respectively, where $A_{p}(\rho), A_{z}(\rho)$ and $A_{+}(\rho)$ are the critical amplitudes (see Table 1 ), and $\psi \simeq 1, \zeta \simeq 1 / 2$ and $\eta_{+} \simeq 1$ are the critical exponents (see Table 2). Figures 2 (a)-(c) display $p^{*} \equiv p / A_{p}(\rho), \Delta z^{*} \equiv \Delta z / A_{z}(\rho)$ and $g_{+}^{*} \equiv g_{+} / A_{+}(\rho)$, respectively. Since $\langle\delta\rangle \sim \Delta \phi$, these results confirm $p \sim \Delta \phi, \Delta z \sim \Delta \phi^{1 / 2}, g_{+} \sim \Delta \phi^{-1}$ and $g_{+}\langle\delta\rangle \approx$ const $[4,5,6,7]$.

From our simulations, we also find $\delta_{\mathrm{m}}$ scales as

$$
\delta_{\mathrm{m}}=A_{\mathrm{m}}(\rho)\langle\delta\rangle^{\lambda}
$$

with the critical amplitude $A_{\mathrm{m}}(\rho)$ (see Table 1 ). Figure 2 (d) shows $\delta_{\mathrm{m}}^{*} \equiv \delta_{\mathrm{m}} / A_{\mathrm{m}}(\rho)$, where we find $\lambda \simeq 1$ similar to $\mu$ (see Table 2). The ratios $A_{\mathrm{m}}(\rho) / B_{\delta}(\rho)$ are not constant (see Table 1), which means that the probability distribution functions of the overlap are not self-similar, but change shape with increasing $\rho$ [25]. 
(a)

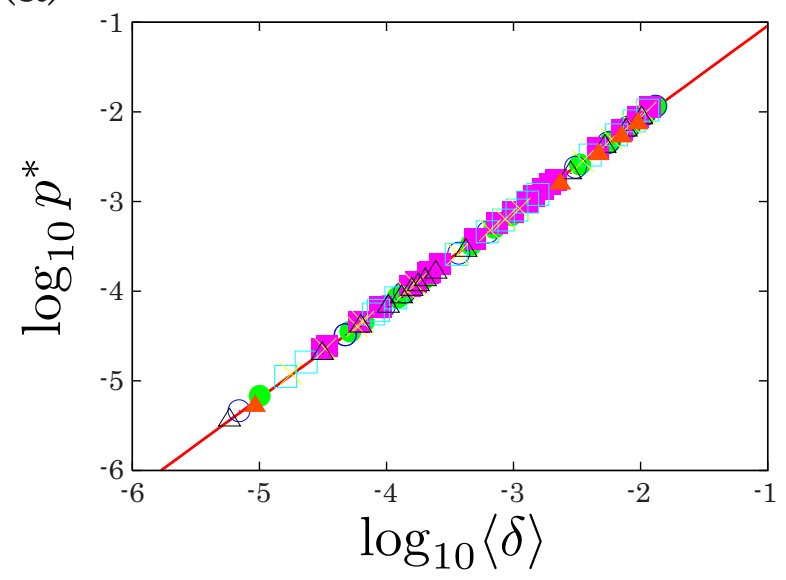

(c)

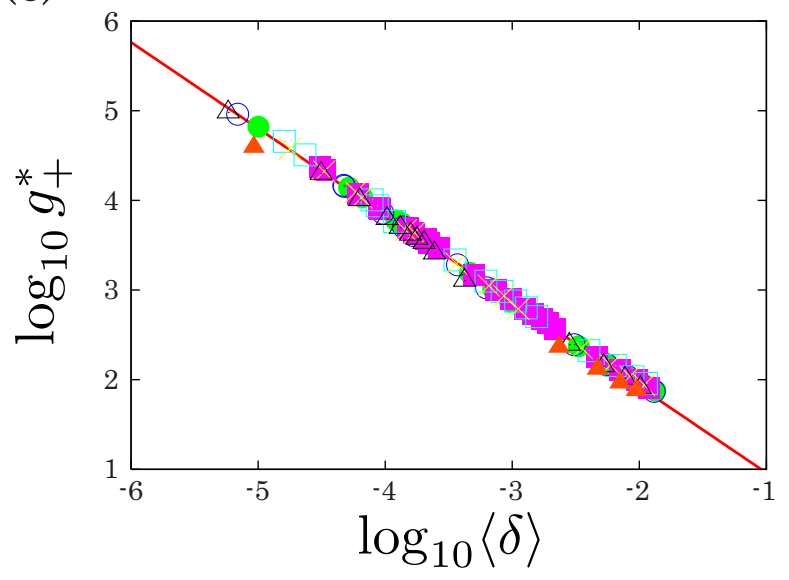

(b)

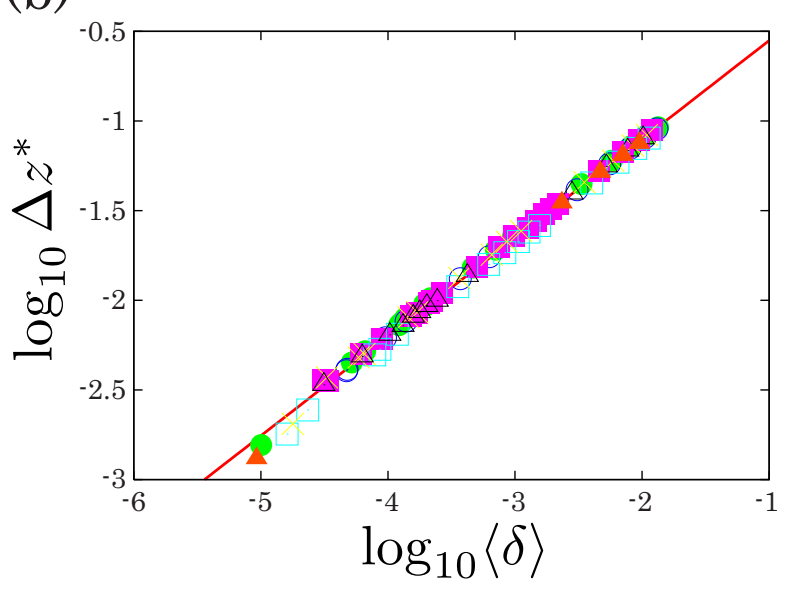

(d)

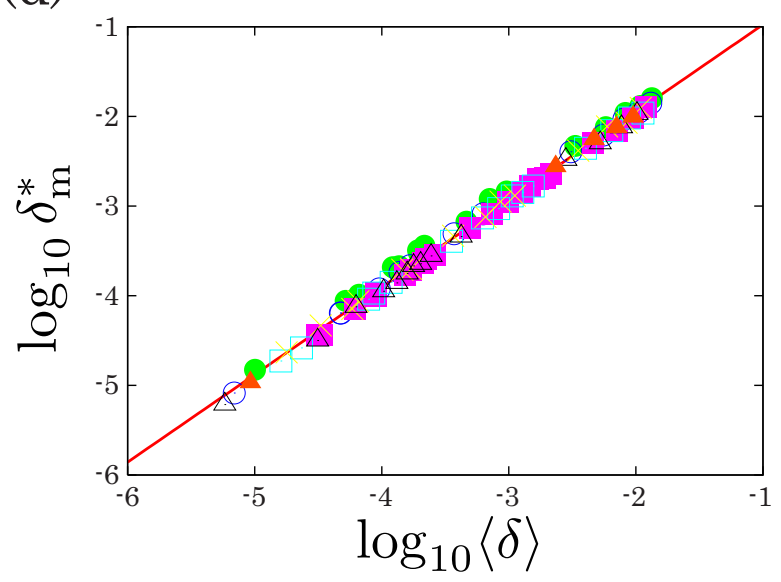

FIGURE 2. (Color online) Double logarithmic plots of (a) $p^{*}$, (b) $z^{*}$, (c) $g_{+}^{*}$ and (d) $\delta_{\mathrm{m}}^{*}$ as functions of $\langle\delta\rangle$. The solid circles, open circles, solid squares, open squares, crosses, open triangles and solid triangles are the data for $\rho=1.2,1.4,1.6,1.8,2.0,2.2$ and 2.4 , respectively.

TABLE 2. Estimated exponents and errors.

\begin{tabular}{cccccc}
\hline function & base & exponent & value & deviation & error [\%] \\
\hline$\langle\delta\rangle$ & $\Delta \phi$ & $\mu$ & 1.006 & \pm 0.003 & 0.349 \\
$p$ & $\langle\delta\rangle$ & $\psi$ & 1.039 & \pm 0.001 & 0.065 \\
$\Delta z$ & $\langle\delta\rangle$ & $\zeta$ & 0.551 & \pm 0.001 & 0.124 \\
$g_{+}$ & $\langle\delta\rangle$ & $\eta_{+}$ & 0.960 & \pm 0.001 & 0.148 \\
$\delta_{\mathrm{m}}$ & $\langle\delta\rangle$ & $\lambda$ & 0.976 & \pm 0.001 & 0.164 \\
\hline
\end{tabular}

\section{CRITICAL SLOWING DOWN NEAR JAMMING}

After we stop to increase the diameter of each particle at $t=t_{0}$, the system relaxes to its static state. To quantify the relaxation dynamics, we introduce a dimensionless energy

$$
\chi(t) \equiv \frac{K(t) / U(t)}{K\left(t_{0}\right) / U\left(t_{0}\right)},
$$

where $K(t)$ and $U(t)$ are the kinetic and potential energies, respectively. The ratio $K(t) / U(t)$ can be used to estimate $\phi_{c}$, because $U(t)$ drops to zero and $K(t) / U(t)$ diverges if $\phi<\phi_{c}$ [26]. The function $\chi(t)$ is the ratio $K(t) / U(t)$ scaled 
(a)

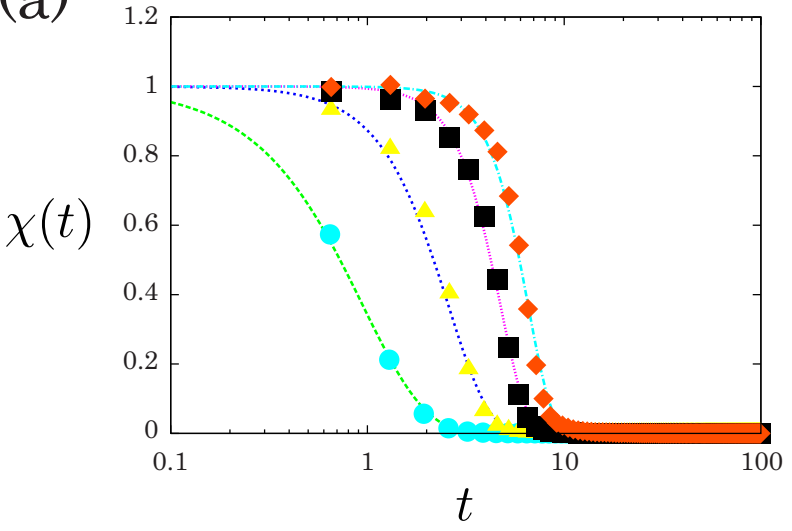

(b)

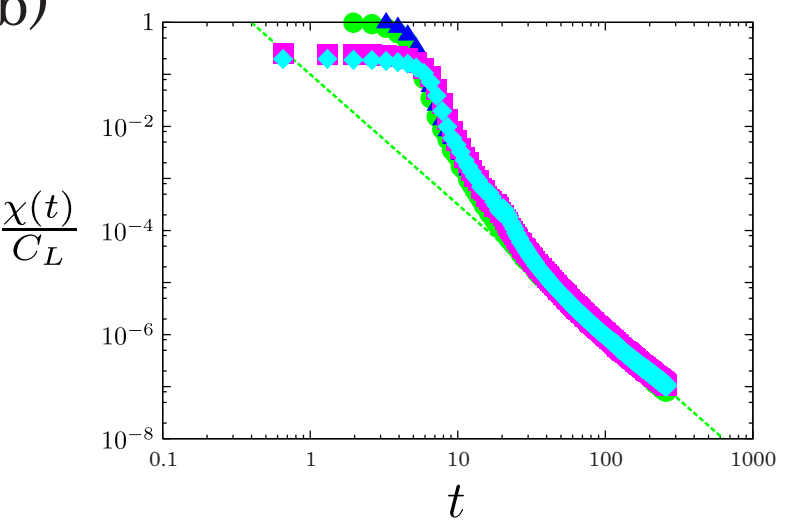

FIGURE 3. (Color online) (a) $\chi(t)$ against logarithmic time above $\phi_{c}$, where the circles, triangles, squares and diamonds are the results of simulations with $\Delta \phi=1.2 \times 10^{-2}, 2.5 \times 10^{-3}, 4.4 \times 10^{-4}$ and $1.4 \times 10^{-4}$, respectively. The dotted lines represent Eq. (11). (b) Double logarithmic plot of $\chi(t) / C_{L}$, where the circles, triangles, squares and diamonds are the results of simulations with $\Delta \phi=5.5 \times 10^{-4}, 4.5 \times 10^{-4}, 2.5 \times 10^{-4}$ and $1.5 \times 10^{-4}$, respectively. The dotted line represents the power law decay with the exponent 2.541. Here, we used $\rho=1.4$.

(a)

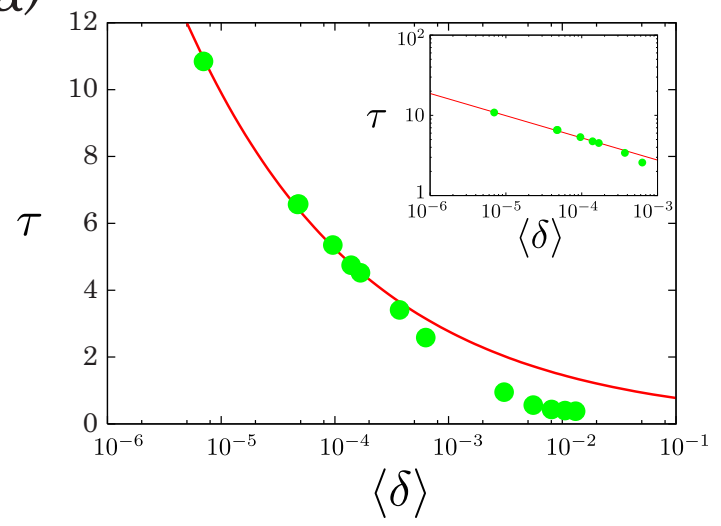

(b)

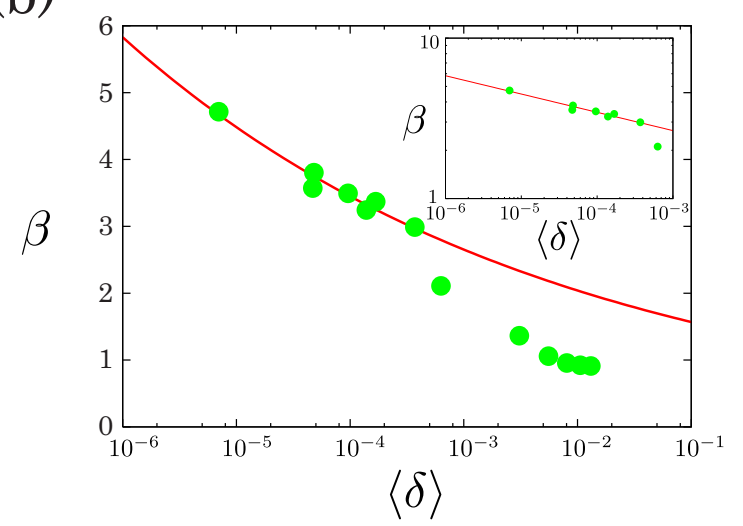

FIGURE 4. (Color online) (a) $\tau$ and (b) $\beta$ against logarithmic $\langle\delta\rangle$, where the insets show the double logarithmic plots of them. The solid circles are our simulation results and the solid lines represent $\tau \propto\langle\delta\rangle^{-\kappa}$ and $\beta \propto\langle\delta\rangle^{-v}$ with $\kappa=0.274 \pm 0.006$ and $v=0.112 \pm 0.008$, respectively. Here, we used $\rho=1.4$.

by the value at $t_{0}$. In the following, we redefine $t_{0}=0$ for simplicity and study the case of $\rho=1.4$.

Figure 3(a) shows our results of $\chi(t)$ against logarithmic time, where the decrease of $\chi(t)$ drastically slows down near the jamming point. To quantify the slowing down, we fit $\chi(t)$ by a stretched exponential function

$$
\chi(t)=e^{-(t / \tau)^{\beta}},
$$

where $\tau$ and $\beta$ are the relaxation time and the stretching exponent, respectively. Figure 4 displays both quantities as functions of $\langle\delta\rangle$. Slightly above $\phi_{c}\left(0<\Delta \phi<10^{-3}\right)$, both $\tau$ and $\beta$ scale as $\tau \propto\langle\delta\rangle^{-\kappa}$ and $\beta \propto\langle\delta\rangle^{-v}$ with $\kappa \simeq 0.27$ and $v \simeq 0.11$, respectively. However, given the rather narrow range of $\tau$ and $\beta$, power laws with such small exponents are not of significance and require a wider range before conclusions can be drawn. It should be noted that $\beta>1$ near the jamming point and $\chi(t)$ decays faster than exponential with time.

Figure 3(b) is a double logarithmic plot of the long term asymptotic behavior of $\chi(t)$ near the jamming point, where we find a power law decay

$$
\chi(t)=C_{L} t^{-\alpha} .
$$


The exponent $\alpha \simeq 2.5$ is not significantly changed by changing $\phi$ and faster than the decay rate of the kinetic energy in the homogeneous cooling state of granular gases, where the exponent is given by 2 [27].

\section{SUMMARY}

In summary, we numerically investigated the static and dynamic properties of two-dimensional bidisperse particles near the jamming transition and systematically studied the dependency of the critical exponents and amplitudes on the size ratio $\rho$. For different size ratios, we found different scaling prefactors of the average and maximum overlaps confirming their different behaviors and thus indicating different shapes of the overlap probability distribution functions [25].

Concerning the dynamics, we used the energy ratio $\chi(t)$ to quantify the relaxation of kinetic energy and report that it resembles a stretched exponential and drastically slows down near the jamming point. The asymptotic behavior of $\chi(t)$ resembles a power law decay with exponent 2.5 , which is faster than the decay rate of the kinetic energy in the homogeneous cooling state of granular gases.

The cases of monodisperse and polydisperse particles are left to future study, as is the case for three dimensions. Although the number of particles in our simulations may be large enough to study the critical area fraction and the critical scaling, we have to investigate the influence of the system size, the growth rate and the restitution coefficient elsewhere.

\section{ACKNOWLEDGMENTS}

We thank M. van Hecke, B. Tighe, H. Hayakawa, T. Hatano, N. V. Brilliantov and K. Yazdchi for fruitful discussions and T. Weinhart for his critical reading. This work was financially supported by the NWO-STW VICI grant 10828.

\section{REFERENCES}

1. A. J. Liu, and S. R. Nagel, Nature (London) 396, 21-22 (1998).

2. V. Trappe, V. Prasad, L. Cipelletti, P. N. Segre, and D. A. Weitz, Nature 411, 772-775 (2001).

3. C. Song, P. Wang, and H. A. Makse, Nature 453, 629-632 (2008).

4. C. S. O'Hern, S. A. Langer, A. J. Liu, and S. R. Nagel, Phys. Rev. Lett. 88, 075507 pp. 1-4 (2002).

5. C. S. O'Hern, L. E. Silbert, A. J. Liu, and S. R. Nagel, Phys. Rev. E 68, 011306 pp. 1-19 (2003).

6. $\quad$ T. S. Majmudar, M. Sperl, S. Luding, and R. P. Behringer, Phys. Rev. Lett. 98, 058001 pp. 1-4 (2007).

7. M. van Hecke, J. Phys.: Condens. Matter 22, 033101 pp. 1-24 (2010).

8. J. Brujić, S. F. Edwards, D. V. Grinev, I. Hopkinson, D. Brujić, and H. A. Makse, Faraday Discuss. 123, $207-220$ (2003).

9. H. P. Zhang, and H. A. Makse, Phys. Rev. E 72, 011301 pp. 1-12 (2005).

10. F. Bolton, and D. Weaire, Phys. Rev. Lett. 65, 3449-3451 (1990).

11. D. J. Durian, Phys. Rev. Lett. 75, 4780-4783 (1995).

12. S. Alexander, Phys. Rep. 296, 65-236 (1998).

13. L. E. Silbert, D. Ertaş, G. S. Grest, T. C. Halsey, and D. Levine, Phys. Rev. E 65, 031304 pp. 1-6 (2002).

14. L. E. Silbert, A. J. Liu, and S. R. Nagel, Phys. Rev. E 73, 041304 pp. 1-8 (2006).

15. A. Donev, S. Torquato, and F. H. Stillinger, Phys. Rev. E 71, 011105 pp. 1-14 (2005).

16. X. Cheng, Phys. Rev. E 81, 031301 pp. 1-12 (2010).

17. B. D. Lubachevsky, and F. H. Stillinger, J. Stat. Phys. 60, 561-583 (1990).

18. G. Prisi, and F. Zamponi, Rev. Mod. Phys. 82, 789-845 (2010).

19. A. Donev, S. Torquato, F. H. Stillinger, and R. Connelly, J. App. Phys. 95, 989-999 (2004).

20. S. Luding, and E. Bauer, Geomechanics and Geotechnics: From Micro to Macro 1, 495-499 (2011).

21. S. Luding, J. Phys.: Condens. Matter 17, S2623-S2640 (2005).

22. S. Luding, Advances in Complex Systems 4, 379-388 (2001).

23. V. Ogarko, and S. Luding, J. Chem. Phys. 136, 124508 pp. 1-11 (2012).

24. M. P. Allen, and D. J. Tildesley, Computer Simulation of Liquids, Oxford University Press, New York, 1987.

25. M. K. Müller, T. Pöschel, and S. Luding, Chem. Phys. 375, 600-605 (2010).

26. F. Göncü, O. Duran, and S. Luding, AIP Conf. Procs. "Powders and Grains 2009" 1145, 531-534 (2009).

27. S. Luding, Nonlinearity 22, R101-R146 (2009). 